\title{
Global bifurcation of the nondifferentiable Sturm-Liouville problems with indefinite weight and spectral parameter in the boundary condition
}

\author{
Ulkar V.Gurbanova
}

Received: 09.04.2020 / Revised: 15.09.2020/ Accepted: 16.11.2020

Abstract. In this paper, we consider the nondifferentiable Sturm-Liouville problem with an indefinite weight function and a spectral parameter in the boundary condition. The global bifurcation of solutions to this problem from zero is studied. The existence of four families of unbounded continua of solutions corresponding to the usual nodal properties and bifurcation from the intervals of the line of trivial solutions is proved.

Keywords. nondifferentiable Sturm-Liouville problem, indefinite weight function, spectral parameter in the boundary condition, bifurcation point, global bifurcation

Mathematics Subject Classification (2010): 34B09, 34B15, 34C10, 34C23, 34K11, 34L20, 34K18, 47J10, 47J15.

\section{Introduction}

We consider the nonlinear Sturm-Liouville problem

$$
\begin{gathered}
\ell y \equiv-\left(p(x) y^{\prime}\right)^{\prime}+q(x) y=\lambda r(x) y+h\left(x, y, y^{\prime}, \lambda\right), x \in(0,1), \\
b_{0} y(0)=d_{0} p(0) y^{\prime}(0), \\
\left(a_{1} \lambda+b_{1}\right) y(1)=p(1) y^{\prime}(1),
\end{gathered}
$$

where

(i) $p \in C^{1}[0,1], q, r \in C[0,1], p>0, q \geq 0$ and $r$ changes sign on $[0,1]$,

(ii) $b_{0}, d_{0}, a_{1}, b_{1}$ are real constants such that

$$
\left|b_{0}\right|+\left|d_{0}\right|>0, b_{0} d_{0} \geq 0 \text { and, if } b_{0}=0 \text {, then } q \not \equiv 0 \text {, and } a_{1}>0, b_{1} \leq 0 \text {. }
$$

(iii) the nonlinear term $h$ has the representation $h=f+g$, where $f$ and $g$ are continuous on $[0,1] \times \mathbb{R}^{3}$, satisfying the following conditions:

$$
u g(x, u, s, 0) \leq 0, u f(x, u, s, 0) \leq 0,(x, u, s) \in[0,1] \times \mathbb{R}^{2} ;
$$

U.V. Gurbanova

Ganja State University, Ganja, Azerbaijan

E-mail: ulya1812dok2@mail.ru 


$$
\left|\frac{f(x, u, s, \lambda)}{u}\right| \leq M,(x, u, s, \lambda) \in[0,1] \times \mathbb{R}^{3}, 0<|u| \leq 1,|s| \leq 1 ;
$$

where $M>0$ is some constant;

$$
g(x, u, s, \lambda)=o(|u|+|s|) \text { as }|u|+|s| \rightarrow 0,
$$

uniformly in $(x, \lambda) \in[0,1] \times \Lambda$, for any bounded interval $\Lambda \subset \mathbb{R}$.

In recent years, the study of bifurcation of nonlinear eigenvalue problems for differential equations has been intensively developed. This is due to the fact that problems of this type arise in the study of various processes of natural science (see $[2,12,15,22]$ and the bibliography therein). Note that the problem (1.1)-(1.3) with $a_{1}=0$ describes the selectionmigration process in population genetics (see $[12,15])$.

The global bifurcation of non-trivial solutions of nonlinear eigenvalue problems for ordinary differential equations of second and fourth order, and partial differential equations with definite weight function were studied in detail in $[1,2,4,8,9,11,26-28]$. These problems in cases where the weighting functions are indefinite have been studied in recent times in works $[3,5-7,17,18,23,25]$. In these works, the existence of two or fourth families of unbounded continua that bifurcate from points and intervals of lines of trivial solutions are proved. It was also established that these continua also possess oscillatory properties of the corresponding linear problems.

Global bifurcation of solutions of the nonlinear eigenvalue problem (1.1)-(1.3) with $f \equiv$ 0 was studied in recent paper [19], where it was shown that there exists fourth families of unbounded continua of solutions corresponding to the usual nodal properties and bifurcating from points (the first components of which are eigenvalues of linear problem (1.1)-(1.3) with $f, g \equiv 0$ ) of the line of trivial solutions.

In this paper, we study the global bifurcation from zero of nontrivial solutions of the nonlinear problem (1.1)-(1.3).

\section{Operator treatment of the boundary value problem (1.1)-(1.3) and some required results}

Let $H=L_{2}(0,1) \oplus \mathbb{C}$ be a Hilbert space equipped with the scalar product

$$
(\hat{y}, \hat{v})_{H}=(\{y, \alpha\},\{v, \beta\})=(y, v)_{L_{2}}+a_{1}^{-1} \alpha \bar{\beta},
$$

where $(y, v)_{L_{2}}=\int_{0}^{1} y(x) \overline{v(x)} d x$.

We define a linear operator $A$ in $H$ acting according to the rule

$$
A \hat{y}=A\{y, \alpha\}=\left\{\ell(y), p(1) y^{\prime}(1)-b_{1} y(1)\right\},
$$

on the domain

$$
\begin{aligned}
D(A)=\left\{\hat{y}=\{y, \alpha\} \in H: y, p y^{\prime}\right. & \in A C[0,1], \ell(y) \in L_{2}(0,1), \\
b_{0} y(0) & \left.=d_{0} p(0) y^{\prime}(0), \alpha=a_{1} y(1)\right\} .
\end{aligned}
$$

It follows from [16] that $A$ is a self-adjoint operator in $H$. Moreover, by [19, Lemma 2.1] (see also [10, Lemma 2.1]) this operator is positive definite on $D(A)$. Then, due to the maximum-minimum properties of the eigenvalues (see [13, Ch. 6, §1, paragraph 4]), we have

$$
(A \hat{y}, \hat{y})_{H} \geq \lambda_{1}(\hat{y}, \hat{y})_{H}, \hat{y} \in D(A),
$$

where $\lambda_{1}$ ia a first eigenvalue of the operator $A$. 
Now we define a multiplication operator $R: H \rightarrow H$, and nonlinear operators $F, G$ : $\mathbb{R} \times D(A) \rightarrow H$ by

$$
\begin{gathered}
R \hat{y}=R\{y, \alpha\}=\{r y, \alpha\}, \\
F(\lambda, \hat{y})=F\{y, \alpha\}=\left\{f\left(\cdot, y, y^{\prime}, \lambda\right), \alpha\right\}, \\
G(\lambda, \hat{y})=G\{y, \alpha\}=\left\{f\left(\cdot, y, y^{\prime}, \lambda\right), \alpha\right\},
\end{gathered}
$$

where $\alpha=a_{1} y^{\prime}(1)$. Thus the nonlinear eigenvalue problem (1.1)-(1.3) can be rewritten in the following equivalent operator equation

$$
A \hat{y}=\lambda R \hat{y}+F(\lambda, \hat{y})+G(\lambda, \hat{y}), \hat{y} \in D(A),
$$

and consequently, between the solutions $(\lambda, y)$ and $(\lambda, \hat{y})$ of problems (1.1)-(1.3) and (2.3) there is a one-to-one correspondence

$$
(\lambda, y) \leftrightarrow(\lambda, \hat{y}), \hat{y}=\{y, \alpha\}, \alpha=a_{1} y(1) .
$$

It is obvious that the linear eigenvalue problem

$$
A \hat{y}=\lambda R \hat{y}, \hat{y} \in D(A),
$$

is equivalent to the following Sturm-Liouville eigenvalue problem

$$
\begin{gathered}
(\ell y)(x)=\lambda r(x) y(x), x \in(0,1), \\
b_{0} y(0) \stackrel{=}{=} d_{0} p(0) y^{\prime}(0),\left(a_{1} \lambda+b_{1}\right) y(1)=p(1) y^{\prime}(1) .
\end{gathered}
$$

which obtained from (1.1)-(1.3) by setting $h \equiv 0$. By virtue of [10, Theorem 3.2] (see also $[20,21]$ ) the spectrum of the linear eigenvalue problem $(2.5)$ (or of the pair $\{A, R\}$ of operators $A$ and $R$ ) is discrete and consists of two infinite sequences of real and simple eigenvalues $\lambda_{n \pm}, n=1,2, \ldots$ such that

$$
\begin{aligned}
& 0<\lambda_{1}^{+}<\lambda_{2}^{+}<\ldots<\lambda_{n}^{+}<\ldots, \\
& 0>\lambda_{1}^{-}>\lambda_{2}^{-}>\ldots>\lambda_{n}^{-}>\ldots .
\end{aligned}
$$

Moreover, for each $n \in \mathbb{N}$ and each $\sigma \in\{+,-\}$ the eigenfunction $y_{n}^{\sigma}(x)$, corresponding to the eigenvalue $\lambda_{n}^{\sigma}$, have exactly $n-1$ simple zeros in the interval $(0,1)$.

Denote:qqq

$$
b c_{0}=\left\{y \in C^{1}[0,1]: b_{0} y(0)=d_{0} y^{\prime}(0)\right\} .
$$

Let $E=C^{1}[0,1] \cap b c_{0}$ be the Banach space with the usual norm

$$
\|y\|_{1}=\|y\|_{\infty}+\left\|y^{\prime}\right\|_{\infty},\|y\|_{\infty}=\max _{x \in[0,1]}|y(x)|,
$$

and let $\hat{E}=E \oplus \mathbb{C}$ be the Banach space with the norm

$$
\|\hat{y}\|_{1}=\|\{y, \alpha\}\|_{1}=\|y\|_{1}+|\alpha| .
$$
that

If $\{y, \alpha\} \in D(A)$, then we have $y^{\prime} \in A C[0,1]$ in view of $p \in C^{1}[0,1]$. Hence it follows

$$
y \in C^{1}[0,1] \text {, and } D(A) \subseteq \hat{E} .
$$

Now let $C^{0}[0,1] \oplus \mathbb{R}$ be the Banach space with the norm

$$
\|\hat{y}\|_{0}=\|\{y, \alpha\}\|_{0}=\|y\|_{0}+|\alpha|,\|y\|_{0}=\max _{x \in[0,1]}|y(x)| .
$$

It follows from the definition of the operators $R, F$ and $G$ that

$$
R: \hat{E} \rightarrow C^{0}[0,1] \oplus \mathbb{C}, G: \mathbb{R} \times \hat{E} \rightarrow C^{0}[0,1] \oplus \mathbb{C}, G: \mathbb{R} \times \hat{E} \rightarrow C^{0}[0,1] \oplus \mathbb{C}
$$


Since $\lambda_{1}>0$ it follows from [9, Lemma 3.3] that there exists

$$
\mathcal{A}=A^{-1}: C^{0}[0,1] \oplus \mathbb{C} \rightarrow D(A),
$$

and is a compact and continuous map.

Let the operators $\mathcal{R}: \hat{E} \rightarrow \hat{E}, \mathcal{F}: \mathbb{R} \times \hat{E} \rightarrow \hat{E}$ and $\mathcal{G}: \mathbb{R} \times \hat{E} \rightarrow \hat{E}$ are defined as follows:

$$
\mathcal{R}=\mathcal{A} R, \mathcal{F}=\mathcal{A F} \text { and } \mathcal{G}=\mathcal{A} G,
$$

respectively. Since operator $\mathcal{A}$ is a completely continuous, and the operators $R, F$ and $G$ are continuous and bounded it follows that the operators $\mathcal{R}, \mathcal{F}$ and $\mathcal{G}$ are completely continuous. Moreover, by (1.6) the operator $\mathcal{G}$ satisfy the condition

$$
\mathcal{G}(\lambda, \hat{y})=o\left(\|\hat{y}\|_{1}\right) \text { as }\|\hat{y}\|_{1} \rightarrow 0
$$

uniformly in $\lambda \in \Lambda$ (see [19]).

Thus the nonlinear eigenvalue problem (2.3) (also problem (1.1)-(1.3)) reduces to the following equivalent problem

$$
\hat{y}=\lambda \mathcal{R} \hat{y}+\mathcal{F}(\lambda, \hat{y})+\mathcal{G}(\lambda, \hat{y})
$$

Let $f \equiv 0$ in (1.1). Then (1.1)-(1.3) reduces to the problem

$$
\hat{y}=\lambda \mathcal{R} \hat{y}+\mathcal{G}(\lambda, \hat{y})
$$

By (2.7) it follows from [22, Ch. $4, \S 2$, Theorem 2.1] that problem (2.9) is linearizable, and the linearization of this problem at $\hat{y}=\hat{0}=\{0,0\}$ is given as follows:

$$
\hat{y}=\lambda \mathcal{R} \hat{y}
$$

The linear spectral problem (2.10) is equivalent to the linear eigenvalue problems (2.5) and (2.6).

Let $S_{n}^{\sigma, \nu}$ and $\hat{S}_{n}^{\sigma, \nu}, n \in \mathbb{N}, \sigma \in\{+,-\}, \nu \in\{+,-\}$, be the sets that are defined in $\S 3$ of [19].

In [19] for the linearizable problems (2.9) and (1.1)-(1.3) with $f \equiv 0$ the following global results are established.

Theorem 2.1 For each $n \in \mathbb{N}$, each $\sigma \in\{+,-\}$ and each $\nu \in\{+,-\}$ there exists a continuum $\hat{\mathcal{C}}_{k}^{\sigma, \nu}$ of solutions of problem (2.9) which contains $\left(\lambda_{n}^{\sigma}, \hat{0}\right)$ is contained in $\left(\mathbb{R}^{\sigma} \times\right.$ $\left.\hat{S}_{n}^{\sigma, \nu}\right) \cup\left\{\left(\lambda_{n}^{\sigma}, \hat{0}\right)\right\}$ and is unbounded in $\mathbb{R} \times \hat{E}$, where $\mathbb{R}^{\sigma}=\{z \in \mathbb{R}: \sigma z \in(0,+\infty)\}$.

Theorem 2.2 For each $n \in \mathbb{N}$, each $\sigma \in\{+,-\}$ and each $\nu \in\{+,-\}$ there exists a continuum $\mathcal{C}_{k}^{\sigma, \nu}$ of solutions of problem (1.1)-(1.3) for $f \equiv 0$ which contains $\left(\lambda_{n}^{\sigma}, 0\right)$ is contained in $\left(\mathbb{R}^{\sigma} \times S_{n}^{\sigma, \nu}\right) \cup\left\{\left(\lambda_{n}^{\sigma}, 0\right)\right\}$ and is unbounded in $\mathbb{R} \times E$. 
3 Global bifurcation of solutions of the nondifferentiable problem (1.1)-(1.3)

To study the global bifurcation of solutions to problem (1.1)-(1.3), we consider the following perturbed linear spectral problem

$$
\begin{gathered}
(\ell y)(x)+\psi(x) y(x)=\lambda r(x) y(x), x \in(0,1), \\
b_{0} y(0)=d_{0} p(0) y^{\prime}(0),\left(a_{1} \lambda+b_{1}\right) y(1)=p(1) y^{\prime}(1) .
\end{gathered}
$$

where $\psi(x) \in C[0,1]$ and $\psi(x) \geq 0, x \in[0,1]$. Again, by [10, Theorem 3.2] all the eigenvalues of problem (3) are real real and simple, and form an two infinite increasing sequences $\lambda_{n, \psi}^{ \pm}, n=1,2, \ldots$, such that

$$
\begin{aligned}
& 0<\lambda_{1 \psi}^{+}<\lambda_{2, \psi}^{+}<\ldots<\lambda_{n, \psi}^{+}<\ldots, \\
& 0>\lambda_{1, \psi}^{-}>\lambda_{2, \psi}^{-}>\ldots>\lambda_{n, \psi}^{-}>\ldots ;
\end{aligned}
$$

for each $n \in \mathbb{N}$ and each $\sigma \in\{+,-\}$ the eigenfunction $y_{n, \psi}^{\sigma}(x)$, corresponding to the eigenvalue $\lambda_{n, \psi}^{\sigma}$, have exactly $n-1$ simple zeros in the interval $(0,1)$.

Lemma 3.1 For each $n \in \mathbb{N}$ the following relations hold:

$$
\begin{aligned}
& \lambda_{n}^{+} \leq \lambda_{n, \psi}^{+} \leq \lambda_{n}^{+}\left(1+\frac{K}{\lambda_{1}}\right), \\
& \lambda_{n}^{-}\left(1+\frac{K}{\lambda_{1}}\right) \leq \lambda_{n, \psi}^{-} \leq \lambda_{n}^{-},
\end{aligned}
$$

where

$$
K=\sup \{\psi(x): x \in[0,1]\} .
$$

Proof. Note that the spectral problem reduces to the following equivalent linear eigenvalue problem

$$
A \hat{y}+L_{\psi} \hat{y}=\lambda R \hat{y}, \hat{y} \in D(A),
$$

where the operator $L_{\psi}: H \rightarrow H$ is defined by

$$
L_{\psi} \hat{y}=L_{\psi}\{y, \alpha\}=\{\psi y, 0\} .
$$

Since $A$ is self-adjoint and positive definite operator on $H$ with compact resolvent it follows that $\hat{R}=A^{-\frac{1}{2}} R A^{-\frac{1}{2}}$ and $\hat{L}_{\psi}=A^{-\frac{1}{2}} L_{\psi} A^{-\frac{1}{2}}$ are self-adjoint and compact on $H$.

Let $\tilde{\lambda}$ be an eigenvalue of (3.4) and $\tilde{\hat{y}}$ the corresponding eigenvector, and $\tilde{\hat{v}}=A^{\frac{1}{2}} \tilde{\hat{y}}$. Then $(\tilde{\lambda}, \tilde{\hat{y}})$ solves the following linear problem

$$
\hat{v}+\hat{L}_{\psi} \hat{v}=\lambda \hat{R} \hat{v} .
$$

Since all eigenvalues of (3.4) are nonzero it follows from (3.5) that $\tilde{\lambda}$ is an eigenvalue of problem

$$
\left(\hat{R}-\frac{1}{\tilde{\lambda}} \tilde{L}_{\psi}\right) \hat{v}=\frac{1}{\lambda} \hat{v}
$$

Therefore, $\frac{1}{\lambda_{k, \psi}^{+}}\left(\frac{1}{\lambda_{k, \psi}^{-}}\right)$is the $k$-th positive (negative) eigenvalue of problem (3.6) for $\tilde{\lambda}=$ $\lambda_{k, \psi}^{+}\left(\tilde{\lambda}=\lambda_{k, \psi}^{-}\right)$. 
By virtue of the min-max (max-min) properties of the eigenvalues of self-adjoint compact operators we have

$$
\begin{aligned}
\lambda_{n, \psi}^{+} & =\min _{V^{(n-1)} \subset H} \max _{\hat{y} \perp V^{(n-1)}} \frac{(\hat{R} \hat{y}, \hat{y})_{H}-\frac{1}{\lambda_{n, \psi}^{+}}\left(\hat{L}_{\psi} \hat{y}, \hat{y}\right)_{H}}{(\hat{y}, \hat{y})_{H}}, \\
\lambda_{n, \psi}^{-} & =\max _{V^{(n-1)} \subset H} \min _{\hat{y} \perp V^{(n-1)}} \frac{(\hat{R} \hat{y}, \hat{y})_{H}-\frac{1}{\lambda_{n, \psi}^{-}}\left(\hat{L}_{\psi} \hat{y}, \hat{y}\right)_{H}}{(\hat{y}, \hat{y})_{H}},
\end{aligned}
$$

where $V^{j}, j \in \mathbb{N}$, is the set of all $j$-dimensional lineals of the space $H$ (see [14]).

It follows from (2.2) that

$$
\left(A^{-\frac{1}{2}} \hat{y}, A^{-\frac{1}{2}} \hat{y}\right)_{H}=\left(A^{-1} \hat{y}, \hat{y}\right)_{H} \leq \frac{1}{\lambda_{1}}(\hat{y}, \hat{y})_{H} .
$$

In view (3.9), by (2.1) we have

$$
\begin{aligned}
&\left(\hat{L}_{\psi} \hat{y}, \hat{y}\right)_{H}=\left(A^{-\frac{1}{2}} L_{\psi} A^{-\frac{1}{2}} \hat{y}, \hat{y}\right)_{H}=\left(L_{\psi} A^{-\frac{1}{2}} \hat{y}, A^{-\frac{1}{2}} \hat{y}\right)_{H} \\
& \leq K\left(A^{-\frac{1}{2}} \hat{y}, A^{-\frac{1}{2}} \hat{y}\right)_{H} \leq \frac{K}{\lambda_{1}}(\hat{y}, \hat{y})_{H},
\end{aligned}
$$

Taking into account (3.10) we have the following relations

$$
\begin{aligned}
& \frac{(\hat{R} \hat{y}, \hat{y})_{H}}{(\hat{y}, \hat{y})_{H}}-\frac{K}{\lambda_{1} \lambda_{n, \psi}^{+}} \leq \frac{(\hat{R} \hat{y}, \hat{y})_{H}-\frac{1}{\lambda_{n, \psi}^{+}}\left(\hat{L}_{\psi} \hat{y}, \hat{y}\right)_{H}}{(\hat{y}, \hat{y})_{H}} \leq \frac{(\hat{R} \hat{y}, \hat{y})_{H}}{(\hat{y}, \hat{y})_{H}}, \\
& \frac{(\hat{R} \hat{y}, \hat{y})_{H}}{(\hat{y}, \hat{y})_{H}}-\frac{K}{\lambda_{1} \lambda_{n, \psi}^{-}} \geq \frac{(\hat{R} \hat{y}, \hat{y})_{H}-\frac{1}{\lambda_{n, \psi}^{-}}\left(\hat{L}_{\psi} \hat{y}, \hat{y}\right)_{H}}{(\hat{y}, \hat{y})_{H}} \geq \frac{(\hat{R} \hat{y}, \hat{y})_{H}}{(\hat{y}, \hat{y})_{H}} .
\end{aligned}
$$

Considering the fact that $\lambda_{n}^{\sigma}=\left.\lambda_{n, \psi}^{\sigma}\right|_{\psi \equiv 0}, \sigma \in\{+,-\}$, by (3.7) and (3.8) from (3.11) and (3.12) we get

$$
\begin{aligned}
& \frac{1}{\lambda_{n}^{+}}-\frac{K}{\lambda_{1} \lambda_{n, \psi}^{+}} \leq \frac{1}{\lambda_{n, \psi}^{+}} \leq \frac{1}{\lambda_{n}^{+}}, \\
& \frac{1}{\lambda_{n}^{-}}-\frac{K}{\lambda_{1} \lambda_{n, \psi}^{-}} \geq \frac{1}{\lambda_{n, \psi}^{-}} \geq \frac{1}{\lambda_{n}^{-}},
\end{aligned}
$$

respectively. Consequently, it follows from (3.13) and (3.14) that

$$
\begin{gathered}
0 \leq \lambda_{n, \psi}^{+}-\lambda_{n}^{+} \leq \frac{K \lambda_{n}^{+}}{\lambda_{1}}, \\
0 \leq \lambda_{n}^{-}-\lambda_{n, \psi}^{-} \leq-\frac{K \lambda_{n}^{-}}{\lambda_{1}},
\end{gathered}
$$

respectively. The relations (3.15) and (3.16) are equivalent (3.2) and (3.3) respectively. The proof of this lemma is complete.

Lemma 3.2 If $(\lambda, y), y \not \equiv 0$, solves the nonlinear problem (1.1)-(1.3), then $\lambda \neq 0$. 
Proof. Let $(0, y)$ be a nontrivial solution of problem (1.1)-(1.3). Then it follows from (2.3) that

$$
A \hat{y}=F(0, \hat{y})+G(0, \hat{y}),
$$

where $\hat{y}=\{y, \alpha\}, \alpha=a_{1} y(1)$. Scalarly multiplying both sides of (3.17) by $\hat{y}$ we obtain

$$
(A \hat{y}, \hat{y})_{H}=(F(0, \hat{y}), \hat{y})_{H}+(G(0, \hat{y}), \hat{y})_{H} \text {. }
$$

which implies that

$$
(F(0, \hat{y}), \hat{y})_{H}+(G(0, \hat{y}), \hat{y})_{H}>0
$$

in view of (2.2).

On the other hand by (2.1) and (1.5) we have

$$
\begin{gathered}
(F(0, \hat{y}), \hat{y})_{H}+(G(0, \hat{y}), \hat{y})_{H}= \\
\int_{0}^{1} f\left(x, y(x), y^{\prime}(x), 0\right) y(x) d x+\int_{0}^{1} g\left(x, y(x), y^{\prime}(x), 0\right) y(x) d x \leq 0,
\end{gathered}
$$

in contradiction with the relation (3.19). The proof of this lemma is complete.

For each $n \in \mathbb{N}$ let

$$
J_{n}^{+}=\left[\lambda_{n}^{+}, \lambda_{n}^{+}+d_{n}^{+}\right], J_{n}^{-}=\left[\lambda_{n}^{+}-d_{n}^{-}, \lambda_{n}^{-}\right],
$$

where

$$
d_{n}^{+}=\frac{M \lambda_{n}^{+}}{\lambda_{1}}, d_{n}^{-}=-\frac{M \lambda_{n}^{-}}{\lambda_{1}} .
$$

Lemma 3.3 For each $n \in \mathbb{N}$, each $\sigma \in\{+,-\}$, each $\nu \in\{+,-\}$, and any sufficiently small $\tau>0$ there exists solution $\left(\lambda_{n, \tau}^{\sigma, \nu}, y_{n, \tau}^{\sigma, \nu}\right) \in \mathbb{R} \times E$ of problem (1.1)-(1.3) such that

$$
\sigma \lambda_{n, \tau}^{\sigma, \nu}>0, y_{n, \tau}^{\sigma, \nu} \in S_{n}^{\sigma, \nu} \text { and }\left\|y_{n, \tau}^{\sigma, \nu}\right\|_{1}=\tau .
$$

Proof. We consider the following approximating problem

$$
\begin{gathered}
\ell y=\lambda r(x) y+f\left(x,|y|^{\varepsilon} y, y^{\prime}, \lambda\right)+g\left(x, y, y^{\prime}, \lambda\right), 0<x<1, \\
b_{0} y(0)=d_{0} p(0) y^{\prime}(0),\left(a_{1} \lambda+b_{1}\right) y(1)=p(1) y^{\prime}(1),
\end{gathered}
$$

where $\varepsilon \in(0,1]$.

We will prove the lemma in two stapes.

Step 1. Let $\tau_{0}$ be the sufficiently small fixed number. Let us show that for each $n \in \mathbb{N}$ there exists $\tau_{n}, 0<\tau_{n}<\tau_{0}$, such that for any $\varepsilon \in(0,1)$ there is no solution $(\mu, w)$ of problem (3.20) satisfying the following conditions:

$$
\operatorname{dist}\left\{\mu, J_{n}^{\sigma}\right\}>\tau_{0}, w \in S_{n}^{\sigma, \nu}, \sigma, \nu \in\{+,-\}, \text { and }\|w\|_{1}<\tau_{n} .
$$

Indeed, otherwise there exists $\varepsilon_{0}>0$ such that problem (3.20) with $\varepsilon=\varepsilon_{0}$ has nontrivial solution $\left(\mu_{\tau}, w_{\tau}\right)$ for any $\tau \in\left(0, \tau_{0}\right)$ that satisfies conditions

$$
\operatorname{dist}\left\{\mu_{\tau}, J_{n}^{\sigma}\right\}>\tau_{0}, w_{\tau} \in S_{n}^{\sigma, \nu}, \sigma, \nu \in\{+,-\} \text {, and }\left\|w_{\tau}\right\|_{1}<\tau \text {. }
$$

Consequently, for any $k \in \mathbb{N}$ there exists a nontrivial solution $\left(\mu_{k}, w_{k}\right)$ of (3.20) with $\varepsilon=\varepsilon_{0}$ such that

$$
\operatorname{dist}\left\{\mu_{k}, J_{n}^{\sigma}\right\}>\tau_{0}, w_{k} \in S_{n}^{\sigma, \nu}, \sigma, \nu \in\{+,-\} \text {, and }\left\|w_{k}\right\|_{1}<\frac{1}{k} .
$$


For each $k \in \mathbb{N}$ we define the function $\psi_{k}(x)$ as follows:

$\psi_{k}(x)=-f\left(x,\left|w_{k}(x)\right|^{\varepsilon_{0}} w_{k}(x), w_{k}^{\prime}(x), \mu_{k}\right) / w_{k}(x)$, if $x \in[0,1], w_{k}(x) \neq 0$, $\psi_{k}(x)=0$ otherwise.

Then $\left(\mu_{k}, w_{k}\right), k \in \mathbb{N}$, is a solution of the following linearizable problem

$$
\begin{aligned}
& \ell y+\psi_{k}(x) y=\lambda r(x) y+g\left(x, y, y^{\prime}, \lambda\right), 0<x<1, \\
& b_{0} y(0)=d_{0} p(0) y^{\prime}(0),\left(a_{1} \lambda+b_{1}\right) y(1)=p(1) y^{\prime}(1) .
\end{aligned}
$$

By Theorem 2.2 for sufficiently $k \in \mathbb{N}$ we can choose a sufficiently small $\tau_{k} \in\left(0, \tau_{0}\right)$ such that

$$
\left|\mu_{k}-\lambda_{k, n}\right|<\tau_{k},
$$

where $\lambda_{k, n}$ is the $n$th eigenvalue of the linear spectral problem (3.22) with $g \equiv 0$. By (1.4) it follows from (3.21) that

$$
0 \leq \psi_{k}(x) \leq M, x \in[0,1]
$$

and consequently, by relations (3.2) and (3.3) we get

$$
\lambda_{k, n} \in J_{n}^{\sigma} .
$$

Then it follows from (3.23) and (3.24) that

$$
\operatorname{dist}\left\{\mu_{k}, J_{n}^{\sigma}\right\}<\tau_{k}<\tau_{0},
$$

which contradicts the condition dist $\left\{\mu_{k}, J_{n}^{\sigma}\right\}>\tau_{0}$.

Step 2. By Step 1, from Theorem 2.2 we obtain that for each $n \in \mathbb{N}$, each $\sigma \in\{+,+,-\}$, each $\mu \in\{+,-\}$ and any $\tau \in\left(0, \tau_{0}\right)$ there exists a nontrivial solution $\left(\lambda_{n, \tau, \varepsilon}^{\sigma, \nu}, y_{n, \tau, \varepsilon}^{\sigma, \nu}\right) \in$ $\mathbb{R} \times E$ of problem (3.20) for any $\varepsilon \in(0,1)$ such that

$$
\operatorname{dist}\left\{\lambda_{n, \tau, \varepsilon}^{\sigma, \nu}, J_{n}^{\sigma}\right\}<\tau_{0}, \sigma \lambda_{n, \tau, \varepsilon}^{\sigma, \nu}>0, y_{n, \tau, \varepsilon}^{\sigma, \nu} \in S_{n}^{\sigma, \nu} \text { and }\left\|y_{n, \tau, \varepsilon}^{\sigma, \nu}\right\|_{1}=\tau \text {. }
$$

Then, in view of (1.5) and (1.6), by the Arzela-Ascoli theorem it follows form (3.20) that the set

$$
\left\{\left(\lambda_{n, \tau, \varepsilon}^{\sigma, \nu}, y_{n, \tau, \varepsilon}^{\sigma, \nu}\right): \varepsilon \in(0,1)\right\} \in \mathbb{R} \times E
$$

is precompact. Hence from any positive sequence $\left\{\varepsilon_{k}\right\}_{k=1}^{\infty}$ converging to 0 one can choose a subsequence $\left\{\varepsilon_{k_{m}}\right\}_{m=1}^{\infty}$ such that

$$
\left(\lambda_{n, \tau, \varepsilon_{k_{m}}}^{\sigma, \nu}, y_{n, \tau, \varepsilon_{k_{m}}}^{\sigma, \nu}\right) \rightarrow\left(\lambda_{n, \tau}^{\sigma, \nu}, y_{n, \tau}^{\sigma, \nu}\right) \text { as } m \rightarrow \infty .
$$

Putting $(\lambda, y)=\left(\lambda_{n, \tau, \varepsilon_{k_{m}}}^{\sigma, \nu}, y_{n, \tau, \varepsilon_{k_{m}}}^{\sigma, \nu}\right)$ in (3.22) and then passing to the limit as $m \rightarrow \infty$, we obtain that $\left(\lambda_{n, \tau}^{\sigma, \nu}, y_{n, \tau}^{\sigma, \nu}\right)$ solves the boundary value problem (1.1)-(1.3). Note that

$$
y_{n, \tau, \varepsilon}^{\sigma, \nu} \in \overline{S_{n}^{\sigma, \nu}} \text { and }\left\|y_{n, \tau, \varepsilon}^{\sigma, \nu}\right\|_{1}=\tau,
$$

which by [19, Lemma 4.1] implies that $y_{n, \tau, \varepsilon}^{\sigma, \nu} \in S_{n}^{\sigma, \nu}$. Moreover, by Lemma 3.2 it follows that $\sigma \lambda_{n, \tau}^{\sigma, \nu}>0$. The proof of this lemma is complete.

Lemma 3.3 implies the following result.

Corollary 3.1 For each $n \in \mathbb{N}$, each $\sigma \in\{+,-\}$ and each $\nu \in\{+,-\}$ the set of bifurcation points of problem (1.1)-(1.3) with respect to the set $S_{n}^{\sigma, \nu}$ is nonempty. If $(\lambda, 0)$ is a bifurcation point of (1.1)-(1.3) with respect to the set $S_{n}^{\sigma, \nu}$, then $\lambda \in J_{n}^{\sigma}$. 
Using Corollary 3.1, we define the following sets. For each $n \in \mathbb{N}$, each $\sigma \in\{+,-\}$ and each $\nu \in\{+,-\}$ let $\tilde{\mathcal{D}}_{n}^{\sigma, \nu}$ be the union of all the components $\tilde{\mathcal{D}}_{n, \lambda}^{\sigma, \nu}$ of the closure of the set of solutions of problem (1.1)-(1.3) branching from bifurcation points $(\lambda, 0) \in J_{n}^{\sigma, \nu} \times\{0\}$ with respect to the set $\mathbb{R} \times S_{n}^{\sigma, \nu}$. Now let $\mathcal{D}_{n}^{\sigma, \nu}=\tilde{\mathcal{D}}_{n}^{\sigma, \nu} \cup\left(J_{n}^{\sigma, \nu} \times\{0\}\right)$ which is connected in $\mathbb{R} \times E$.

Theorem 3.1 For each $n \in \mathbb{N}$, each $\sigma \in\{+,-\}$ and each $\nu \in\{+,-\}$ the connected set $\mathcal{D}_{n}^{\sigma, \nu}$ is unbounded in $\mathbb{R} \times E$ and contained in $\left(\mathbb{R}^{\sigma} \times S_{n}^{\sigma, \nu}\right) \cup\left\{\left(\lambda_{n, \sigma}, \hat{0}\right)\right\}$.

By virtue of the one-to-one correspondence (2.4) from Theorem 3.1, we obtain the following result.

Theorem 3.2 For each $n \in \mathbb{N}$, each $\sigma \in\{+,-\}$ and each $\nu \in\{+,-\}$ the connected set $\hat{\mathcal{D}}_{n}^{\sigma, \nu}$ is unbounded in $\mathbb{R} \times \hat{E}$ and contained in $\left(\mathbb{R}^{\sigma} \times \hat{S}_{n}^{\sigma, \nu}\right) \cup\left\{\left(\lambda_{n, \sigma}, \hat{0}\right)\right\}$.

Proof of Theorem 3.1. By Lemma 3.2, it follows from the definition of the sets $\mathcal{D}_{n}^{\sigma, \nu}$, $n \in \mathbb{N}, \sigma \in\{+,-\}, \nu \in\{+,-\}$, that

$$
\mathcal{D}_{n}^{\sigma, \nu} \subset \mathbb{R}^{\sigma} \times E .
$$

If $(\lambda, y) \in \mathbb{R} \times \partial S_{n}^{\sigma, \nu}, n \in \mathbb{N}, \sigma \in\{+,-\}, \nu \in\{+,-\}$, then by Lemma 4.1 of [19] we get $y \equiv 0$. Consequently, it follows from the definition of these sets that

$$
\mathcal{D}_{n}^{\sigma, \nu} \subset\left(\mathbb{R}^{\sigma} \times S_{n}^{\sigma, \nu}\right) \cup\left\{\left(\lambda_{n, \sigma}, \hat{0}\right)\right\} .
$$

Next, by Theorem 2.2, Lemmas 3.1-3.3, Corollary 3.1 and an argument similar to that of [24, Theorem 2.1], we can show that $\mathcal{D}_{n}^{\sigma, \nu}$ is unbounded in $\mathbb{R} \times \hat{E}$. The proof of Theorem 3.2 is complete.

\section{References}

1. Aliyev, Z.S.: Some global results for nonlinear fourth order eigenvalue problems, Cent. Eur. J. Math. 12(12), 1811-1828, (2014).

2. Aliyev, Z.S.: On the global bifurcation of solutions of some nonlinear eigenvalue problems for ordinary differential equations of fourth order, Sb. Math. 207(12) 3-29, (2016).

3. Aliyev, Z.S., Huseynova, R.A: Bifurcation in nonlinearizable eigenvalue problems for ordinary differential equations of fourth order with indefinite weight, Electron. J. Qual. Theory Differ. Equat. (2017)(92), 1-12, (2017).

4. Aliyev, Z.S., Mamedova, G.M.: Some global results for nonlinear Sturm-Liouville problems with spectral parameter in the boundary condition, Ann. Polon. Math. 115(1), 75-87, (2015).

5. Aliyev, Z.S., Hasanova, Sh.M.: Global Bifurcation of positive solutions of semi-linear elliptic partial differential equations with indefinite weight, Z. Anal. Anwend. 38(1) 1$15,(2019)$.

6. Aliyev, Z.S., Hasanova, Sh.M.: Global bifurcation of positive solutions from zero in nonlinearizable elliptic problems with indefinite weight, J. Math. Anal. Appl. 491(1) (2020), doi.org/10.1016/j.jmaa.2020.124252.

7. Aliyev, Z.S., Nasirova (Ashurova) L.V.: Bifurcation of positive and negative solutions of nonlinearizable Sturm-Liouville problems with indefinite weight, Math. Notes, Miskolc, 21(1), 19-29, (2020).

8. Berestycki, H: On some nonlinear Sturm-Liouville problems, J. Diffential Equations 26(3) 375-390, (1977). 
9. Binding, P.A., Browne, P.J., Watson, B.A.: Spectral problem for nonlinear Sturm- Liouville equations with eigenparameter dependent boundary conditions, Canad. J. Math. 52(2) 248-264, (2000).

10. Binding, P.A., Browne, P.J., Watson, B.A.: Left definite Sturm-Liouville problems with eigenparameter dependent boundary conditions, Differential Integral Equations 12, 167-182, (1999).

11. Dai, G, Ma, R: Bifurcation from intervals for Sturm-Liouville problems and its applications, Electron. J. Differ. Equ. (2014)(3), 1-10, (2014).

12. Cantrell, R.S., Cosner, C: Spatial Ecology via ReactionDiffusion Equations, Ser. Math. Comput. Biol., John Wiley and Sons Ltd., Chichester, UK (2003).

13. Courant R, Hilbert, D: Methoden der Mathematischen Physik, I, Springer, Berlin, 1931; English transl., Interscience, New York, 1953.

14. Figueiredo, de D.G.: Positive solutions of semilinear elliptic problems, in Differential Equations, Lecture Notes in Math., 957, Springer, Berlin-New York, 34-47, (1982).

15. Fleming, W.H: A selection-migration model in population genetics. J. Math. Biology 2, 219-233, (1975).

16. Fulton, C.T.: Two-point boundary value problems with eigenvalue parameter contained in the boundary conditions, Proc. Roy. Soc. Edinburgh Sect. A 77(3-4) 293-308, (1977).

17. Cano-Casanova, S.: Compact components of positive solutions for superlinear indefinite elliptic problems of mixed type, Topol. Methods Nonlinear Anal. 23(1) 45-72, (2004).

18. Cano-Casanova, S., Lopez-Gomez, J., Molina-Meyer, M: Isolas: compact solution components separated away from a given equilibrium curve, Hiroshima Math. J. 34(2) 177199, (2004).

19. Gurbanova, U.V., Bifurcation in nonlinear Sturm-Liouville problems with indefinite weight and spectral parameter in the boundary condition, 2020, in press.

20. Hess, P., Kato, T: On some linear and nonlinear eigenvalue problems with indefinite weight function, Comm. Partial Differential Equations 5 999-1030, (1980).

21. Ince, E.L.: Ordinary Differential Equations, Berlin Heidelberg: Springer-Verlag, (1924).

22. Krasnoselski, M.A.: Topological methods in the theory of nonlinear integral equations, Macmillan, New York, (1965).

23. Lopez-Gomez, J., Molina-Meyer, M.: Bounded components of positive solutions of abstract fixed point equations: mushrooms, loops and isolas, J. Differential Equations 209(2) 416-441, (2005).

24. Makhmudov A.P., Aliev, Z.S.: Global bifurcation of solutions of certain nonlinearizable eigenvalue problems, Diff.. Equat. 25(1), 71-76, (1989).

25. Nasirova, L.V.: Global bifurcation from intervals of solutions of nonlinear SturmLiouville problems with indefinite weight, Trans. Natl. Acad. Sci. Azerb. Ser. Phys.Tech. Math. Sci. Mathematics 39(4), 148-154, (2019).

26. Rabinowitz, P.H.: Some global results for nonlinear eigenvalue problems, J. Funct. Anal. 7(3), 487-513, (1971).

27. Rynne, B.P.: Bifurcation from zero or infinity in Sturm-Liouville problems which are not linearizable, J. Math. Anal. Appl. 228(1), 141-156, (1998).

28. K. Schmitt, H. L. Smith, On eigenvalue problems for nondifferentiable mappings, $J$. Differential Equations 33(3) 294-319, (1979). 